PHYSICAL REVIEW D 92, 039904(E) (2015)

\title{
Erratum: Bottomonium hyperfine splittings from lattice nonrelativistic QCD including radiative and relativistic corrections [Phys. Rev. D 89, 031502(R) (2014)]
}

\author{
R. J. Dowdall, C. T. H. Davies, T. Hammant, R. R. Horgan, and C. Hughes
}

(Received 4 August 2015; published 26 August 2015)

DOI: 10.1103/PhysRevD.92.039904

PACS numbers: 12.38.Gc, 11.15.Ha, 14.40.Pq, 99.10.Cd

A numerical error in two previous theoretical papers [1,2] means that the coefficients $d_{1}$ and $d_{2}$ of four-quark operator terms used in this paper are changed. The details of this error are given in the corresponding errata to [1,2]. For clarity we reproduce here the whole of the Table I, but only the entries in columns 7 and 8 are changed, giving respectively the coefficients $d_{1} \alpha_{s}^{2}$ and $d_{2} \alpha_{s}^{2}$.

TABLE I. Parameters used for the valence quarks. $a m_{b}$ is the bare $b$ quark mass in lattice units, $u_{0 L}$ is the Landau link. The $c_{i}, d_{i}$ are coefficients of terms in the NRQCD action [see Eq. (1)]. $c_{4}, d_{1}$ and $d_{2}$ use $\alpha_{s}$ in the V scheme at scale $\pi / a$. The other coefficients use different scales as discussed in Ref. [21].

\begin{tabular}{lllllllll}
\hline \hline Set & $a m_{b}$ & $u_{0 L}$ & $c_{1}, c_{6}$ & $c_{5}$ & $c_{4}$ & $d_{1} \alpha_{s}^{2}$ & $d_{2} \alpha_{s}^{2}$ & $\alpha_{s}(\pi / a)$ \\
\hline 1 & 3.31 & 0.8195 & 1.36 & 1.21 & 1.23 & -0.1021 & 0.0306 & 0.275 \\
2 & 2.73 & 0.834 & 1.31 & 1.16 & 1.19 & -0.058 & 0.016 \\
3 & 1.95 & 0.8525 & 1.21 & 1.12 & 1.18 & -0.026 & 0.006 & 0.255 \\
\hline \hline
\end{tabular}

TABLE III. Fit results in lattice units for the $1 S$ and $2 S$ energies of the $\Upsilon$ and $\eta_{b}$ for the $\mathcal{O}\left(v^{6}\right)$ action with and without four-quark interactions.

\begin{tabular}{lcccc}
\hline \hline Set & $a E_{\Upsilon}(1 S)$ & $a E_{\Upsilon}(2 S)$ & $a E_{\eta_{b}}(1 S)$ & $a E_{\eta_{b}}(2 S)$ \\
\hline \multicolumn{4}{c}{$\mathcal{O}\left(v^{6}\right)$ action } \\
1 & $0.27547(6)$ & $0.6917(14)$ & $0.22950(5)$ & $0.6748(12)$ \\
2 & $0.28786(3)$ & $0.6332(9)$ & $0.25148(3)$ & $0.6180(6)$ \\
3 & $0.30269(3)$ & $0.5573(10)$ & $0.27484(2)$ & $0.5461(9)$ \\
& & & \\
1 & $0.27699(6)$ & $\mathcal{O}\left(v^{6}\right)$ action + four-quark interactions & $0.6755(13)$ \\
2 & $0.28850(3)$ & $0.6997(13)$ & $0.22460(5)$ & $0.6166(9)$ \\
3 & $0.30271(2)$ & $0.6340(10)$ & $0.24897(3)$ & $0.5437(5)$ \\
\hline \hline
\end{tabular}

We have redone the calculation for the bottomonium hyperfine splitting using the correct coefficients and we present the revised results in Table III for the $1 S$ and $2 S$ energies of the $\Upsilon$ and $\eta_{b}$ in the case where the four-quark interactions are included. We reproduce the whole of the revised Table III for clarity but only the last three rows are changed. We also give here the revised figures for Figs. 1-3. Table IV gives the revised error budget. This has changed very little, but a typographical error in the row marked "EM effects" is corrected.

In the abstract the results given in the last two sentences should read, respectively, $M_{\Upsilon}(1 S)-M_{\eta_{b}}(1 S)=60.0(6.4) \mathrm{MeV}$ and $\left(M_{\Upsilon}(2 S)-M_{\eta_{b}}(2 S)\right) /\left(M_{\Upsilon}(1 S)-M_{\eta_{b}}(1 S)\right)=0.445(28)$.

On page 1, second column, the sentence starting "Including the ..." should read "Including the corrections gave a hyperfine splitting of 65(9) MeV where ...". In the text below Eq. (4) the sentence beginning "We take Gaussian priors"

\footnotetext{
${ }^{1}$ The hyperfine splitting using the uncorrected values of $d_{1}, d_{2}$ was found to be 70(9) MeV in [21]. We estimate the correction to the hyperfine splitting using a potential model analysis, as done in [21], but using the corrected values of these coefficients. We find the corrected hyperfine splitting to be $65(9) \mathrm{MeV}$, and it is this value that is plotted in Fig. 3.
} 


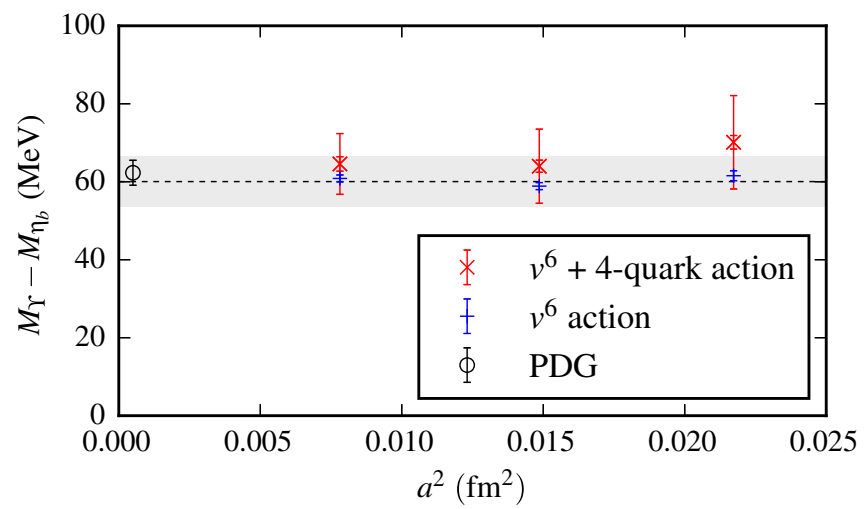

FIG. 1 (color online). The fit result for the $1 S$ hyperfine splitting from the $v^{6}$ action with spin-dependent four-quark operators. The two error bars are as follows: the smaller band includes statistics and scale setting, and the larger band includes correlated errors from missing radiative corrections to $c_{4}, d_{1}, d_{2}$ and quark mass tuning errors. The grey band is our final result, including all systematic errors. Also shown are the pure $v^{6}$ results, which are not included in the fit, and the PDG average [2].

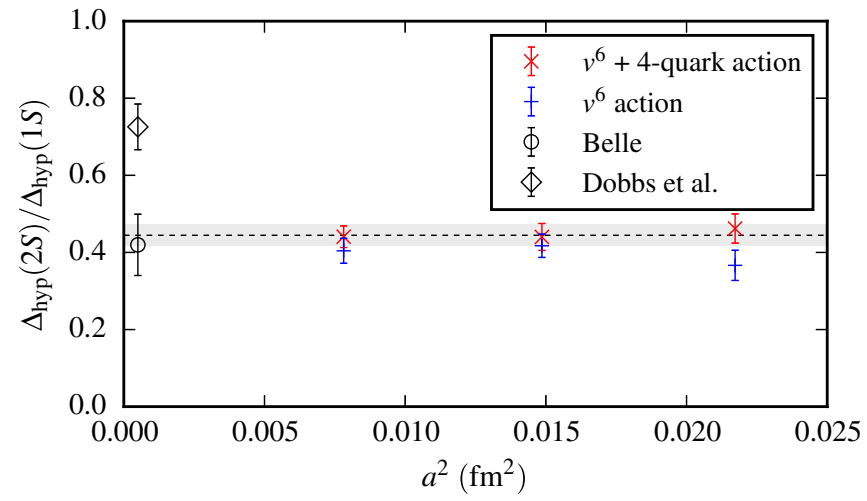

FIG. 2 (color online). The fit result for the hyperfine ratio from the $v^{6}$ action with spin-dependent four-quark operators. Also shown are the pure $v^{6}$ results, which are not included in the fit, and the results from Belle and Dobbs et al. [5, 6].

should read "We take Gaussian priors of mean 0 and width 1 on all the coefficients in the fit to the $1 \mathrm{~S}$ hyperfine splitting. We tighten the prior on $k_{1}$ to $0.0(3)$ in fitting $R_{H}$ since the action includes radiatively improved $a^{2}$ lattice spacing corrections and we also expect cancellation of discretization errors in the ratio."

Equation (5) becomes

$$
\begin{aligned}
\Delta_{\text {hyp }}(1 S) & =60.0(6.4) \mathrm{MeV} \\
\Delta_{\text {hyp }}(2 S) / \Delta_{\text {hyp }}(1 S) & =0.445(28) .
\end{aligned}
$$

On page 5, in Sec. IV, the sentence starting "Our result for the 1S splitting of ..." should be removed.

Equation (6) should read

$$
\Delta_{\text {hyp }}(2 S)=27.7(1.7)_{\text {latt }}(1.4)_{\exp } \mathrm{MeV} \text {. }
$$




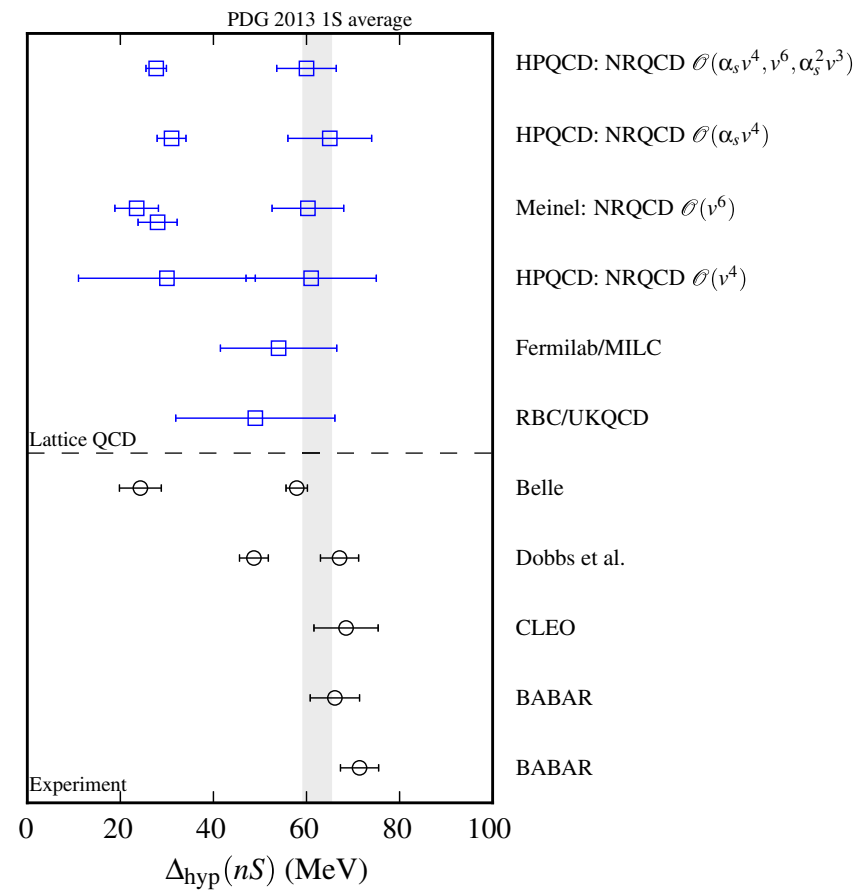

FIG. 3 (color online). Comparison of our result (top) with other lattice calculations [18, 20, 21, 23, 35] and experimental [1-6] results for the hyperfine splittings. The $2 S$ hyperfine splitting is on the left and the $1 S$ on the right. The $1 S$ value plotted for the earlier $\mathcal{O}\left(\alpha_{s} v^{4}\right)$ HPQCD result has been corrected for the $d_{1}, d_{2}$ error and the $2 S$ value uses the 2013 PDG average for $\Delta_{\text {hyp }}(1 S)$ to extract $\Delta_{\text {hyp }}(2 S)$ from the ratio given in that paper. Meinel quotes two results for $\Delta_{\text {hyp }}(2 S)$, normalizing the result using either the $1 S$ hyperfine or the $1 P$ tensor splitting. The results are taken as given in Ref. [23].

TABLE IV. Full error budget for the $1 S$ hyperfine splitting and the $2 S$ to $1 S$ ratio. All errors are in percent.

\begin{tabular}{lrr}
\hline \hline Error $\%$ & $\Delta_{\text {hyp }}(1 S)$ & $R_{H}$ \\
\hline Stats/fitting & 0.2 & 4.1 \\
Uncertainty in $a$ & 2.2 & 0.0 \\
Scale dependence & 1.3 & 2.5 \\
NRQCD $a m_{b}$ dependence & 3.6 & $<0.0$ \\
NRQCD radiative $\alpha_{s} v^{6}$ & 3.7 & 0.0 \\
NRQCD radiative $\alpha_{s}^{2} v^{4}$ in $c_{4}$ & 7.1 & $<0.1$ \\
Statistical error in $c_{4}^{(1)}$ & 3.2 & 1.4 \\
NRQCD relativistic spin $v^{8}$ & 1.0 & 0.5 \\
NRQCD radiative four-quark $\alpha_{s}^{3} v^{3}$ & 3.0 & 1.3 \\
$m_{b}$ tuning & 0.7 & $<0.1$ \\
$m_{1, \text { sea dependence }}$ & $<.5$ \\
EM effects & $<0.1$ & $<0.1$ \\
Total & 0.3 & 6.3 \\
\hline \hline
\end{tabular}

[1] T. C. Hammant, A. G. Hart, G. M. von Hippel, R. R. Horgan, and C. J. Monahan, Phys. Rev. Lett. 107, 112002 (2011).

[2] T. C. Hammant, A. G. Hart, G. M. von Hippel, R. R. Horgan, and C. J. Monahan, Phys. Rev. D 88, 014505 (2013). 